\title{
Time-Dependent Ferrofluid Dynamics in Symmetry Breaking Transverse
}

\author{
Sebastian Altmeyer \\ Max-Planck-Institute for Dynamics and Self-Organization, Göttingen, Germany \\ Email: sebastian_altmeyer@t-online.de
}

Received April 15, 2013; revised May 15, 2013; accepted May 22, 2013

Copyright (C) 2013 Sebastian Altmeyer. This is an open access article distributed under the Creative Commons Attribution License, which permits unrestricted use, distribution, and reproduction in any medium, provided the original work is properly cited.

\begin{abstract}
We investigate the Taylor-Couette flow of a rotating ferrofluid under the influence of symmetry breaking transverse magnetic field in counter-rotating small-aspect-ratio setup. We find only changing the magnetic field strength can drive the dynamics from time-periodic limit-cycle solution to time-independent steady fixed-point solution and vice versa. Thereby both solutions exist in symmetry related offering mode-two symmetry with left- or right-winding characteristics due to finite transverse magnetic field. Furthermore the time-periodic limit-cycle solutions offer alternately stroboscoping both helical left- and right-winding contributions of mode-two symmetry. The Navier-Stokes equations are solved with a second order time splitting method combined with spatial discretization of hybrid finite difference and Galerkin method.
\end{abstract}

Keywords: Taylor-Couette Flow; Ferrofluids; Reynolds Number; Symmetry Breaking; Rotating System and Boundary Layer

\section{Introduction}

Since first study by G. I. Taylor [1], the flow between two concentric differentially rotating cylinders, the socalled Taylor-Couette flow, has been investigated using either theoretical, experimental and numerical approaches and has played a central role in the development of hydrodynamic stability theory [2-5].

Especially in last decades, this simple geometry has become refocused as there has been much increased interest in flows of complexer like magnetic fluids, e.g. ferrofluids [6] which are often used in laboratory experiments to study geophysical flows $[7,8]$.

Ferrofluids [6] are manufactured fluids consisting of dispersions of magnetized nanoparticles in a variety of liquid carriers and are stabilized against agglomeration by the addition of a surfactant monolayer onto the particles. In the absence of an applied magnetic field, the magnetic nanoparticles are randomly orientated, the fluid has zero net magnetization, and the presence of the nanoparticles provides a typically small alteration to the fluids viscosity and density. When a sufficiently strong magnetic field is applied, the ferrofluid flows toward regions of the magnetic field and properties of the fluid such as the viscosity are altered [6,9], and the hydrodynamics of the system can be significantly changed
[10-21]. Till this day most of these works only considered the influence of magnetic fields onto steady, timeindependent flows. Thus there is a lack of, either numerical or experimental, researches for consequences of magnetic fields onto time-dependent flows.

Likewise numerous numerical, theoretical and experimental investigations have shown that the effects of physical end-walls are evident [22-26] even in very long Taylor-Couette systems (large aspect ratio, $\Gamma$ ) and have a significant influence on the flow dynamics. The presence of end-walls, even in the limit of being infinitely far apart completely destroys the axial translation invariance in the idealized theory $[22,23]$ and results in imperfect bifurcation. With only inner cylinder rotating and outer cylinder at rest, the flow dynamics for small systems $\Gamma \approx 1$ is dominated by the competition between several socalled normal and anomalous mode solutions leading to very rich dynamics $[22-24,27,28]$. For very short systems only one or two Taylor cells are present in the annulus $[29,30]$.

In the present paper, we elucidate the influence of a symmetry breaking transverse magnetic field onto the hydrodynamics of counter-rotating ferrofluid with special respect to time-dependent flow. Without magnetic fields such flows have been studied mainly for co-rotating cy- 
linders with the focus on nonlinear-pattern formation [31,32] with an emphasis on magneto-hydrodynamical phenomena with respect to astrophysical application [33$35]$ or even in turbulent flows [36,37]. Thus it is well known that axial magnetic fields can drive turbulence via the magneto-rotational instability [38,39].

This paper is subdivided as follows. Following the introduction, Section 2 describes the mathematical formulation of the problem. Hereafter Section 3 shortly introduces flow state in absence of magnetic field which we have chosen as initial state for further investigation of the magnetic field modifications. Sections 4 and 5 present the main results as the bifurcation scenario with variation of the magnetic field strength and the spatiotemporal behavior of the flows. Finally, Section 6 concludes the main results.

\section{System and Theoretical Description}

Consider an incompressible, isothermal, homogeneous, mono-dispersed ferrofluid with kinematic viscosity $v$ and density $\rho$ within the annular gap of a Taylor-Couette system, consisting of two concentric, independently rotating cylinders (c.f. Figure 1). The inner and outer cylinders of radii $R_{1}$ and $R_{2}$ rotate at angular speeds $\omega_{1}$ and $\omega_{2}$, respectively. The top and bottom end-walls are stationary. They are a distance $\Gamma d$ apart, where $\Gamma$ is the non-dimensional aspect ratio and $d=R_{2}-R_{1}$ is the gap width. The system is described using a cylindrical polar coordinate system $(r, \theta, z)$ with a velocity field $(u, v, w)$.

Either the radius ratio of the cylinders is hold fixed to $R_{1} / R_{2}=0.5$ and the aspect ratio to $\Gamma=1$. We consider homogeneous external fields in transverse $x$-direction of strength $H_{x}$ where $x=r \cos \theta$. Using the gap-width $\mathrm{d}$ as the length scale, the diffusion time $d^{2} / v$ as the time scale, scaling pressure with $\rho v^{2} / d^{2}$, and the magnetic field $\boldsymbol{H}$ and the magnetization $\boldsymbol{M}$ with $\left(\rho / \mu_{0}\right)^{0.5} v / \mathrm{d}\left(\mu_{0}\right.$ is the magnetic constant, i.e. magnetic permeability of free space), the non-dimensional governing equations are

$$
\begin{aligned}
& (\partial t+u \cdot \nabla) u-\nabla^{2} u+\nabla p \\
& =(\boldsymbol{M} \cdot \nabla) \boldsymbol{H}+1 / 2 \nabla \times(\boldsymbol{M} \times \boldsymbol{H}), \\
& \nabla \cdot u=0 .
\end{aligned}
$$

On the cylinder surfaces, we consider no-slip boundary conditions $u\left(r_{1}, \theta, z\right)=\left(0, \operatorname{Re}_{1}, 0\right)$ and

$u\left(r_{2}, \theta, z\right)=\left(0, \operatorname{Re}_{2}, 0\right)$, where the inner and outer Reynolds numbers are $R e_{1}=\omega_{1} r_{1} d / v$ and $R e_{2}=\omega_{2} r_{2} d / v$, where $r_{1}=R_{1} / d$ and $r_{2}=R_{2} / d$ are the non-dimensional inner and outer cylinder radii, respectively. In this paper we will hold the differentially rotations of the cy-linders fixed to $R e_{1}=350$ and $R e_{2}=-500$ which gives a rotation ratio $R e_{2} / R e_{1}=-1.429$. Equation (1) is solved together with an equation that describes the

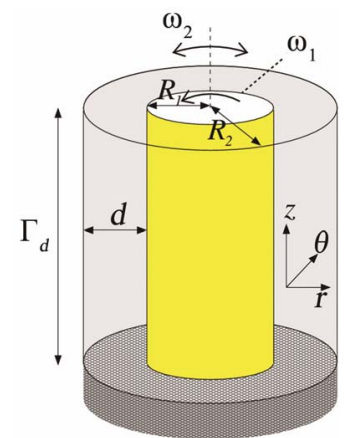

Figure 1. Schematics of the Taylor-Couette system.

magnetization of the ferrofluid. Using the equilibrium magnetization of an unperturbed state with homogeneously magnetized ferrofluid at rest with the mean magnetic moments orientated in the direction of the magnetic field, lead to $\boldsymbol{M}_{e}=\chi \boldsymbol{H}$ (with abbreviation eq for equilibrium). The magnetic susceptibility of the ferrofluid, $\chi$, can be determined by Langevin's formula [40]. The ferrofluid we consider in this paper correspond to APG933 [41] with $\chi=0.9$. Using the near-equilibrium approximation of Niklas [14,15] (small $\left\|\boldsymbol{M}-\boldsymbol{M}_{e}\right\|$ and small relaxation times $\Omega_{\tau} \ll 1$, where $\boldsymbol{\Omega}=\nabla \times u / 2$ is the vorticity ( $\Omega$ gives the absolute value) and $\tau$ is the magnetic relaxation time), as already presented in $[10,19]$.

$$
\boldsymbol{M}-\boldsymbol{M}_{e}=c_{n}^{2} \Omega \times H,
$$

where $c_{n}^{2} \Omega=\tau /\left(1 / \chi+\tau \mu_{0} H^{2} / 6 \mu \Phi\right)$ is the Niklas coefficient, $\mu$ is the dynamic viscosity, and $\Phi$ is the volume fraction of the magnetic material.

Using Equation (2) the magnetization can be eliminated from Equation (1), resulting in the ferrohydrodynamic equation of motion [14]:

$$
\begin{aligned}
& (\partial t+u \cdot \nabla) u-\nabla^{2} u+\nabla p_{m} \\
& =-c_{n}^{2}[\boldsymbol{H} \nabla \cdot \boldsymbol{F}+\boldsymbol{H} \times(\nabla \times \boldsymbol{F}) / 2],
\end{aligned}
$$

where $\boldsymbol{F}=\boldsymbol{\Omega} \times \boldsymbol{H}$ and $p_{m}$ is the dynamic pressure incorporating all magnetic terms which can be written as gradients. Here, we assume that the internal magnetic field is equal to the external imposed magnetic field. It is known as a leading order approximation [19] but is sufficiently good for our here focused numerical investigations of time-dependent ferrofluid flows. Then Equation (3) simplifies to

$$
\begin{aligned}
& (\partial t+u \cdot \nabla) u-\nabla^{2} u+\nabla p_{m} \\
& =-s_{x}^{2} \nabla^{2} u-H \times\left[2 \nabla \times(\Omega \times H)-H \times\left(\nabla^{2} u\right)\right]
\end{aligned}
$$

In this approach, the magnetic field and all the magnetic properties of the ferrofluid influence the velocity field only via the magnetic field parameter

$$
s_{x}=\left[2(2+\chi) /(2+\chi)^{2}-\chi^{2} \eta^{2}\right] H_{x} c_{n},
$$


Note that this is the only parameter that will be changed in this paper, all others will be hold fixed.

Equation (4) (including the continuity equation) is solved with our numerical method G1D3 [10,19], which combines finite-differences in $(r, z)$ with Fourier spectral decomposition in $\theta$ and (explicit) 2nd order time splitting. The variables are written as

$$
f(r, \theta, z, t)=\sum_{m=-m_{\max }}^{m_{\max }} f_{m}(r, z, t) \exp (\operatorname{im} \theta),
$$

where $f$ denotes one of $\{u, v, w, p\}$. For the parameter regimes studied here, $m$ max $=8$ provides adequate accuracy and uniform grids with spacing $\delta r=\delta z=0.05$ and time-steps $\delta t<1 / 3800$ are used. For diagnostic purposes, the complex mode amplitudes $f_{m}(r, t)$ obtained from a Fourier decomposition in the axial direction

$$
f_{m}(r, z, t)=\sum_{n} f_{m n}(r, t) \exp (i n k z)
$$

where $k$ is the axial wavenumber, are evaluated. The Navier-Stokes equations together with the boundary conditions for the finite-length Taylor-Couette system with (classical) fluid confined by end-walls are in variant to rotations about the axis and reflection about the axial mid-height. But with ferrofluid in the annulus and imposed transverse magnetic field $\left(s_{x} \neq 0\right)$ these symmetries are broken and thus the flow is inherently full three-dimensional $[10,19,20]$. Interactions of the magnetic terms in the ferro-hydrodynamic equation result in either an axial downward or upward directed force [42] on the side where the magnetic field enters the bulk, i.e. $\varphi=0$, and an inverse directed force on the opposite side $(\varphi=\pi)$ where the field exits the annulus respectively.

\section{Initial State and Notation}

Following we will short present main characteristics of that time-periodic flow in absence of a magnetic field which we have chosen as initial state for discussion of modifications due to the presence of finite transverse magnetic field.

In order to distinguish the different solutions with and without applied magnetic field we will use the following short abbreviations characterizing the different flows.
Hence we will either use $\mathrm{lc}_{0}$ for the axisymmetric timeperiodic limit-cycle solution with $m=0$ symmetry in absence of a magnetic field and $\mathbf{l c}_{2}$ for the limit-cycle solution with finite applied magnetic field which shows $m=2$ symmetry. The same arguments also hold for the steady time-independent fixed point solutions $\mathrm{fp}_{2}$ and $\mathrm{fp}_{0}$ with and without a magnetic field respectively.

Figure 2 presents spatio-temporal snapshots over one period $\tau$ of our referenced time-periodic (initial) limitcycle solution $1 c_{\circ}$ in absence of a magnetic field. It shows isosurfaces of either the angular momentum $r v$ and of the azimuthal vorticity $\eta$ respectively. $\mathrm{Lc}_{0}$ is axisymmetric (only $m=0$ mode contribution, c.f. Equation (7)) but obviously not reflection symmetric. But there are two symmetries related coexisting limit-cycle solutions that bifurcate out of two steady also non-reflection symmetric states that are symmetry related to each other in similar way. This is the so-called anomalous mode solution [30]. In literature one finds different meanings of this expression. It can describe a flow state with 1) different (mostly odd) number of vortices in the annulus or 2) different flow directions (mostly combined with 1)) near the axial boundaries - the lids. This is classical invert directed (normal mode flow) but also flows with either one or even both outward directed flow exist - the anomalous mode solution. Finally it is also common 3) to describe flows with different size of vortices, i.e. normally in very short systems (as considered here) where one vortex dominates the dynamics and the minor vortex just plays a subsidiary role [29]. Hence the only symmetry relation of these anomalous modes is the time-translation with period $\tau$.This also holds for the herefrom bifurcation limitcycle solution - an existing inverted flow pattern (c.f. online available material movie1.avi and movie2.avi).

\section{Bifurcation Scenario}

As global measure of the flow we use the modal kinetic energy

$$
E:=\sum_{m} E_{m}=\int_{0}^{2 \pi} \int_{0}^{\Gamma} \int_{r_{i}}^{r_{o}} u_{m} u *_{m} r \mathrm{~d} r \mathrm{~d} z \mathrm{~d} \theta
$$

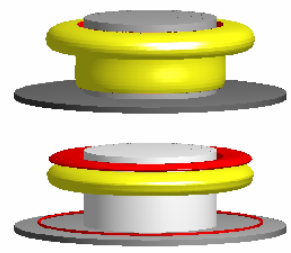

(a)

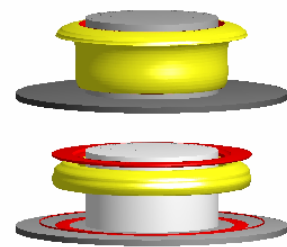

(b)

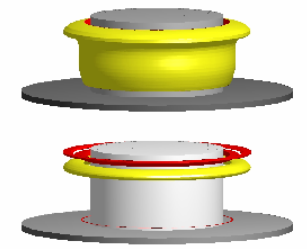

(c)

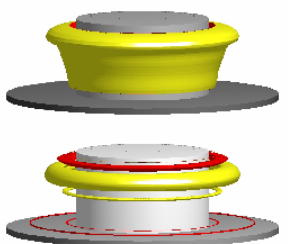

(d)

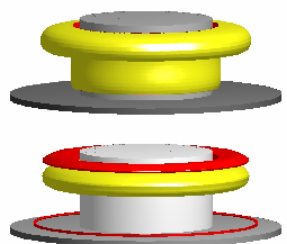

(e)

Figure 2. Isosurfaces of $r v=300$ (top row) and $\eta= \pm 300$ (bottom row) at times $t$ as indicated over one period $(\tau \approx 0.050212)$ for $\mathrm{lc}_{0}$ at $s_{x}=0$ (see also online available material movie1.avi and movie2.avi). (a) $t=0$; (b) $t=\tau / 4$; (c) $t=\tau / 2$; (d) $t=3 \tau / 4$; (e) $t$ $=\tau$. 
where $u_{m} u *_{m}$ is the $m$-th (complex conjugated) Fourier mode of the velocity field. Due to time-dependence of the solutions $\mathrm{lc}_{0}$ and $\mathrm{lc}_{2}$ we will further use the (long-) time-averaged energy $\bar{E}$. Likewise or local measure we also use either Fourier modes of the radial velocity at mid-height and mid-gap

$u_{m n}=u_{m n}(d / 2,0, \Gamma / 2, t)$ (c.f. Equation (7)) and sooner the azimuthal vorticity at two points symmetri- cally displaced about mid-plane on the inner cylinder, $\eta^{-}=\eta\left(r_{1}, 0, \Gamma / 4, t\right)$ and $\eta^{+}=\eta\left(r_{1}, 0,3 \Gamma / 4, t\right)$.

Figure 3 shows the variation with $\mathrm{s} \square$ of time-averaged modal kinetic global energy $\bar{E}$ and for either $m=0$ and $m=2$ modes the peak-to-peak amplitudes $\Delta \mathrm{u}_{01}$ and $\Delta \mathrm{u}_{21}$ together with its corresponding long-time averaged values $\mathrm{u}_{01}$ and $\mathrm{u}_{21}$ respectively. Note that for $s_{x} \approx 0.553$ (below the bifurcation threshold of the $\mathrm{lc}_{2}$ ) the flow $\mathrm{fp}_{2}$ is time-independent. Starting without a magnetic field (left in Figure 3) the initial state is an axisymmetric timeperiodic limit-cycle solution $\mathrm{lc}_{0}$ (c.f. Figure 2) with only $m=0$ mode component. All other azimuthal modes are identical zero. Any finite transverse field component, independent its strength $s_{x}$ destroys this symmetry $[10,11$,

(a)

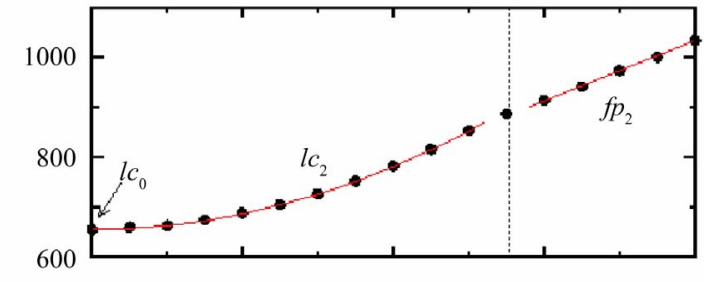

(b)

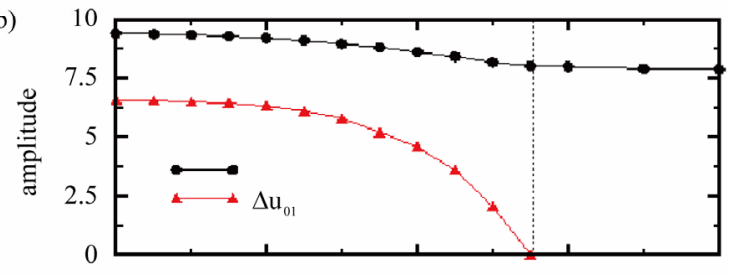

(c)

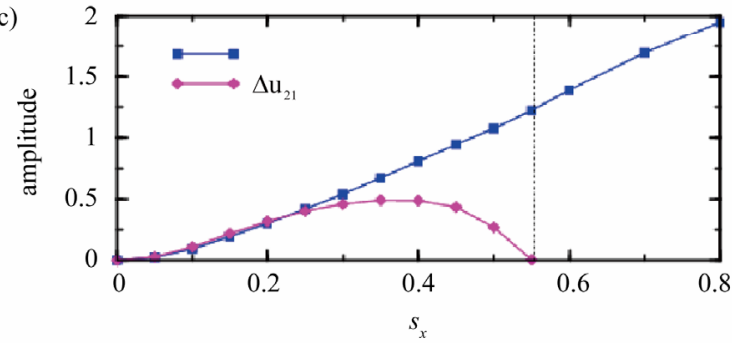

Figure 3. Variation with $s_{x}$ of time-averaged kinetic modal energy $\bar{E}$ (a) and different amplitudes ((b), (c)) at midgap for $l c_{0}, l_{2}$ and $f_{2}$. Shown are either the peak-to-peak amplitudes $\overline{\boldsymbol{u}}_{m n}$ and its long time-averaged values $\overline{\boldsymbol{u}}_{m n}$, respectively, for (b) axisymmetric $(m=0)$ contribution $\left(\Delta u_{01}, \bar{u}_{01}\right)$ and (c) mode-two $(m=2)$ contribution $\left(\Delta u_{21}\right.$, $\bar{u}_{21}$ ) introduced due to finite transverse magnetic field (c.f. Equation (7)). Vertical dotted lines indicate the bifurcation threshold of $l c_{2}$.
19] stimulating $m=2$ contribution. Increasing $s_{x}$ results in enlarging this $m=2$ contribution, which is compensated by decreasing the axisymmetric $m=0$ contribution.

While $\Delta u_{01}$ monotonously decreases with $s_{x} \quad \Delta u_{21}$ firstly increases (up to $s_{x} \approx 0.36$ ) before it also decreases again with $\mathrm{s}_{\mathrm{x}}$. The initially increase and later decrease in $\Delta u_{21}$ results from the contrary competition that larger $s_{x}$ on the one hand side enforce the $m=2$ contribution but simultaneously destabilize the supercritical solution $\mathrm{lc}_{2}$. Finally both vanish at the bifurcation point $s_{x} \approx 0.553$ of $\mathrm{lc}_{2}$ (see dotted lines in Figure 3). Near this bifurcation point, both peak-to-peak amplitudes $\Delta \mathrm{u}_{01}$ and $\Delta u_{21}$ follow a square-root-law indicating the supercritical character of the Hopf bifurcation. Aside the longtime averaged amplitude $\bar{u}_{21}$ increases monotonously with $s_{x}$, independent of the time-characteristics of the solutions. This increase in $\bar{u}_{21}$ is compensated by a monotonous decrease $\bar{u}_{01}$.

For $s_{x} \approx 0.553$ only $\mathrm{fp}_{2}$ remains in the system. This solution corresponds to the anomalous mode solution [29] in absence of a magnetic field. Here it is modified including strong $m=2$ contribution. Hence this solution does not have the axisymmetry of classical anomalous mode solution. Instead it has $m=2$ symmetry (c.f. Figure 4) due to finite $s_{x}$.

The global energy $\bar{E}$ in the system monotonously increases with field strength $s_{x}$. Thereby it first follows a squared law for $\mathrm{lc}_{2}$ until the bifurcation point $\left(s_{x} \approx 0.553\right)$ and below this boundary it grows almost linearly for $\mathrm{fp}_{2}$. Physically the increased energy results from the enlarged complexity in the bulk due to generation of $m=2$ symmetry.

Figure 5 presents the corresponding period of oscillation $\tau$ for $1 c_{2}$ and lco. Starting at the bifurcation point at $s_{x} \approx 0.553$ (almost right in Figure 5) the period $\tau$ becomes finite at onset of $\mathrm{lc}_{2}$ and decreases with decreasing $s_{x}$ whereby the range of variations are relatively small (c.f. values on ordinate). This behavior is quite similar to the bifurcation of classical limit-cycle solution $\mathrm{lc}_{0}$ out of basic state in absence of magnetic fields [28].

We want to mention that there are some experiments $[11,43]$ (but for significant longer system length $\Gamma$, larger than 28) that observed a kind of hysteresis around the onset of supercritical flows. I.e. the critical field strength for the appearing of solutions out of basic state by increasing the field is different (i.e. larger) then that one where the supercritical flows vanish with decreasing field strength.

This behavior can be explained regarding the axial wavenumber $k$. Accompanied with the variation of fieldstrength $s_{x}$ the axial wavenumber $k$ in the flow can also change as there is a competition between different lengthscales at the inner boundary layer which are pre- 


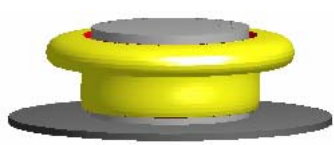

(a)

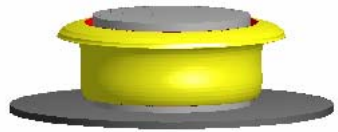

(b)

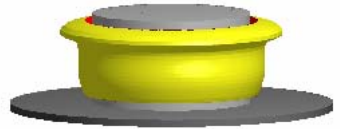

(c)

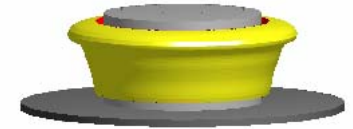

(d)

Figure 4. Isosurfaces of $r v$ and $\eta$ for $\mathrm{fp}_{2}$ at $s_{x}=\mathbf{0 . 6}$ for full and $m=2$ contribution as indicated; isolevel shown at $r v=300$, $[\max ]=[600], r v(m=2)=5,[\max ]=[90], \eta= \pm 300,[\min , \max ]=[-500,500]$ and $\eta(m=2)=180 \&-60),[\min , \max ]=[-250$, 350]. (a) $r v$; (b) $r v(m=2)$; (c) $\eta$; (d) $\eta(m=2)$.

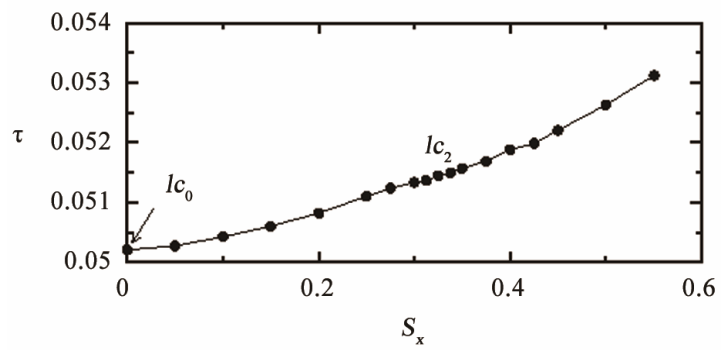

Figure 5. Variation of periodicity length $\tau$ with $s_{x}$ for $\mathrm{lc}_{0}$ and $\mathrm{Ic}_{2}$ (c.f. Figure 3).

ferred by the centrifugal instability due to variation of $\mathrm{Re}_{1}$. Usually flows with different $\mathrm{k}$ have different onsets. But due to the shortness of our here chosen system $(\Gamma=1)$ such kind of effects don't play a role and therefore can be ignored.

Here we want to mention that so far these hysteresis have just been observed for the bifurcation of stationary time-independent flows. The existence of such effects for the bifurcation of time-dependent flows either time-periodic or only quasiperiodic is a still open question that should motivate future experimental work.

\section{Spatio-Temporal Characteristics}

Figure 6 shows time series of flow amplitudes either $u_{01}$ and $u_{21}$ and their corresponding power spectral densities (PSDs) for $\mathrm{lc}_{0}$ with $\left(s_{x}=0\right)$ and $\mathrm{lc}_{2}\left(s_{x}=0.4\right)$ respectively. The inset in the Figure 6(a) illustrates the measure of peak-to-peak amplitude $\Delta$ and its corresponding period length $\tau$ (c.f. Figure 5). Analog to the small variation $\tau$ the PSD also offers minor variations with $s_{x}$ even while the flow loses its axisymmetry due to finite $s_{x}$. The frequencies are only slightly shifted to smaller values with increasing $s_{x}$.

It is well known that magnetic fields with finite transverse component break the axisymmetry due to modetwo coupling $[11,25,28]$. Hence the flow develops two local pinned "bulges" (i.e. $m=2$ symmetry) in azimuth as visible in the isosurface plots of $r v$ and $\eta$ (c.f. Figure $7(\mathbf{c})$ ). The only remaining symmetry for $\mathrm{lc}_{0}$ and $\mathrm{lc}_{2}$ is the time-periodicity.

Note that we also checked the $m=2$ contribution to be the only non-zero component. Starting with random perturbations over all other modes these will die out by time.
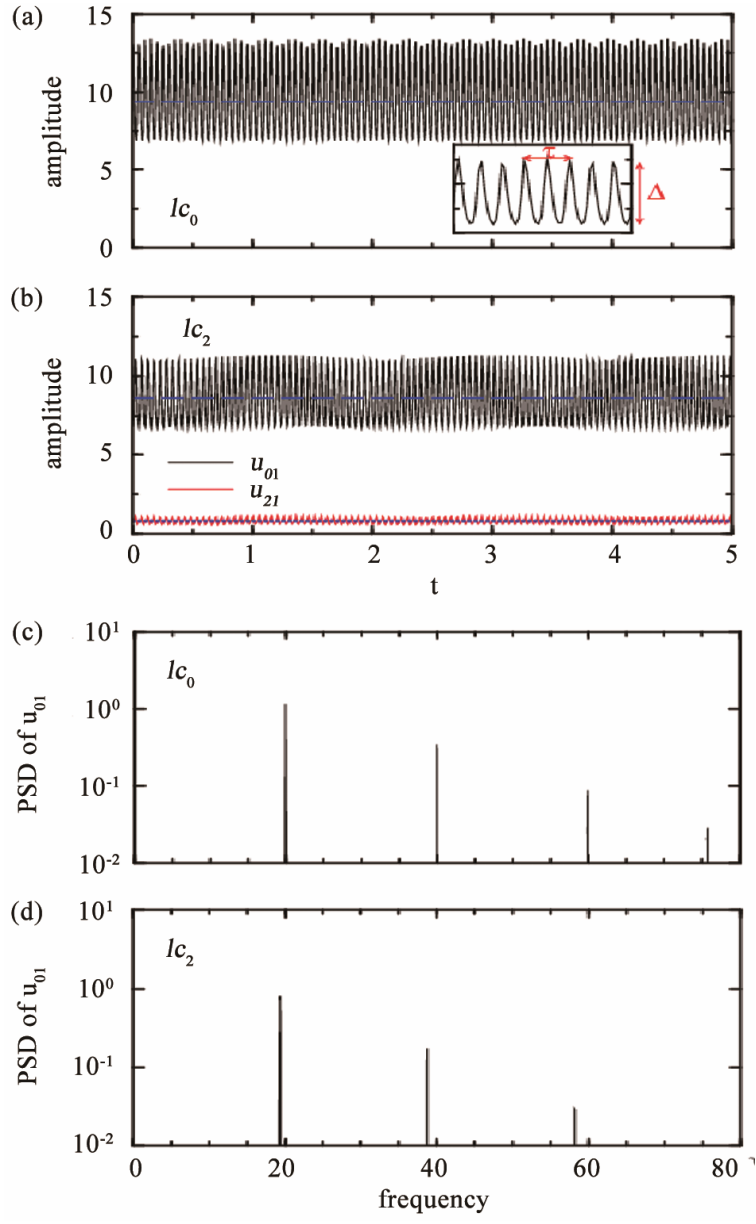

Figure 6. Time series ((a), (b)) of radial flow amplitudes $u_{01}$, $u_{21}$ and its corresponding PSD ((c), (d)) for $\mathrm{Ic}_{0}$ at $s_{x}=0$ and $I c_{2}$ at $s_{x}=0.4$. Horizontal dashed lines in ((a), (b)) indicate long-time averaged values and the inset in (a) illustrates the measure of $\Delta$ and $\tau$.

Comparing the time-dependent flows with and without applied magnetic field one finds significant different characteristics especially in the $m=2$ contribution: 1) Even while the dominant jet oscillating about mid-plane only slightly differs the $m=2$ contribution show a strong time-dependence. It illustrates a kind of stroboscoping over one period whereby the pattern remains localized phase-pinned and non-rotating. 2) The latter stroboscoping in the $m=2$ contribution shows a periodic, alternating change between left- and right-winding shape (c.f. Figures 7(b) and (d)) over one period. This 


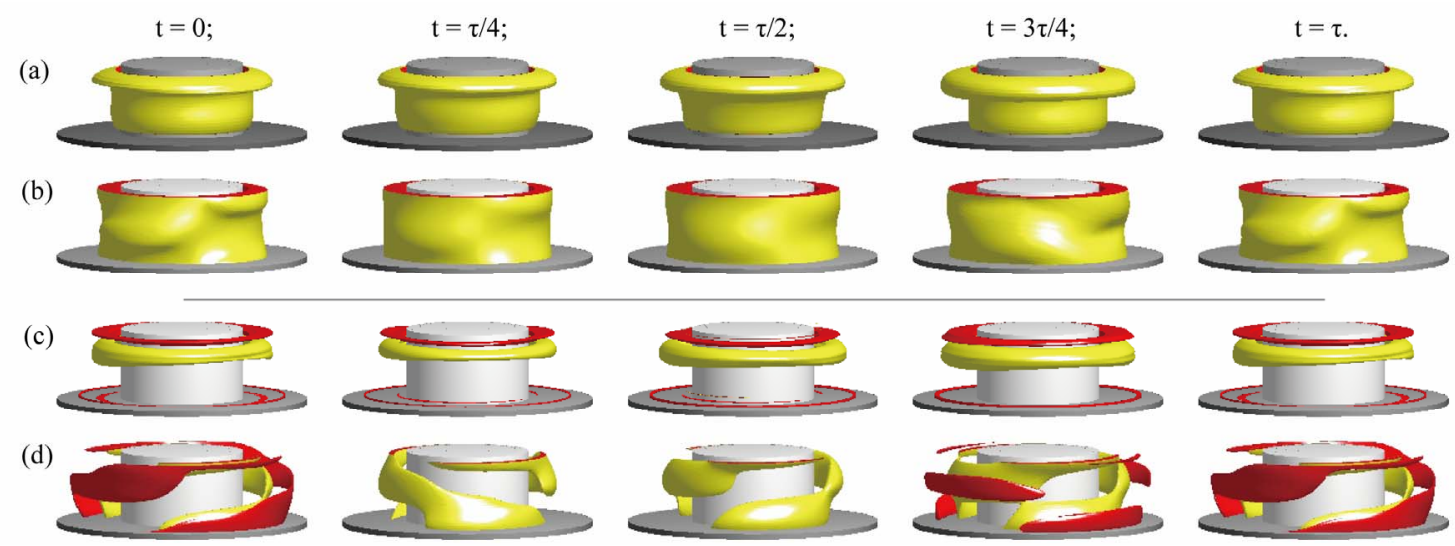

Figure 7. Isosurfaces of $r v((\mathrm{a}),(\mathrm{b}))$ and $\eta((\mathrm{c}),(\mathrm{d}))$ for $\mathrm{lc}_{2}$ at $\boldsymbol{s}_{x}=\mathbf{0 . 4}$ at different times $t$ as indicated over one period $(\tau \approx$ 0.051914); ((a), (c)) show full solution (isolevel shown at $r v=300,[\max ]=[800], \eta= \pm 300,[\min , \max ]=[-600,600])$ and ((b),(d)) $m=2$ contributions (isolevel shown at $r v=50,[\max ]=[250], \eta=180 \&-60$, $[\mathrm{min}, \max ]=[-300,400]$ ) (see online available material movie3.avi, movie4.avi, movie5.avi, and movie6.avi), respectively. Note that even while the solution is time-periodic the $m=2$ contribution do not rotate. It just illustrates a stroboscoping behavior of left- and right-winding characteristics to appear and vanish again over one period.

differs from the shape of $\mathrm{fp}_{2}$ which offers only one (dominant) helicity (left- or right-winding) characteristics in its $m=2$ contribution. But note that the isolevels for $\eta(m=2)$ in Figure 7 are different and therefore can just give a qualitative indentation.

In Figure 4 isosurfaces of $\mathrm{fp}_{2}$ are presented. The pattern is strongly deformed with visible $m=2$ symmetry due to the relative large field strength $s_{x}=0.6$. Even while the surface plot of $r v$ only show small modulations the wavy-like deformation of vortices is obvious. But interestingly the $m=2$ contribution in particular $\eta(m=2)$ do not show any significant helical shape in contrast to the latter discussed pattern for $\mathrm{lc}_{2}$ (c.f. Figure 7).

Figure 8 shows phase portraits of $1 \mathrm{c}_{0}, \mathrm{lc}_{2}$ and $\mathrm{fp}_{2}$ on $\left(\eta^{-}, \eta^{+}\right)$for various $s_{x}$ as indicated. Dotted line with points indicates stationary and time-independent flows.

Starting in $\mathrm{fp}_{2}$ with $s_{x}=0.8$ (top right) and decreasing $s_{x} \quad \mathrm{lc}_{2}$ bifurcates out of the steady state $\mathrm{fp}_{2}$ at $s_{x}$ about 0.553 . Different circles illustrate the evolution of $1 \mathrm{c}_{0}$ and $\mathrm{lc}_{2}$ which are obviously not symmetric here. But note that the symmetry related solution bifurcating in the same way out of the corresponding flow of the opposite anomalous mode which parallel exists.

\section{Momentum Flux and Cross-Flow Energy}

Taking the $\theta$ component of the Navier-Stokes equations and averaging over cylinders at fixed radius $r$ the angular momentum flux [44] can be defined as

$$
J:=r^{3}\left(\langle u v / r\rangle_{a(r)}-v \partial r\langle v / r\rangle_{a(r)}\right)
$$

where $a(r)$ stands for the averaging over the surface of a concentric cylinder at radius $r$. Moreover the longtime mean value can be obtained from an additional average

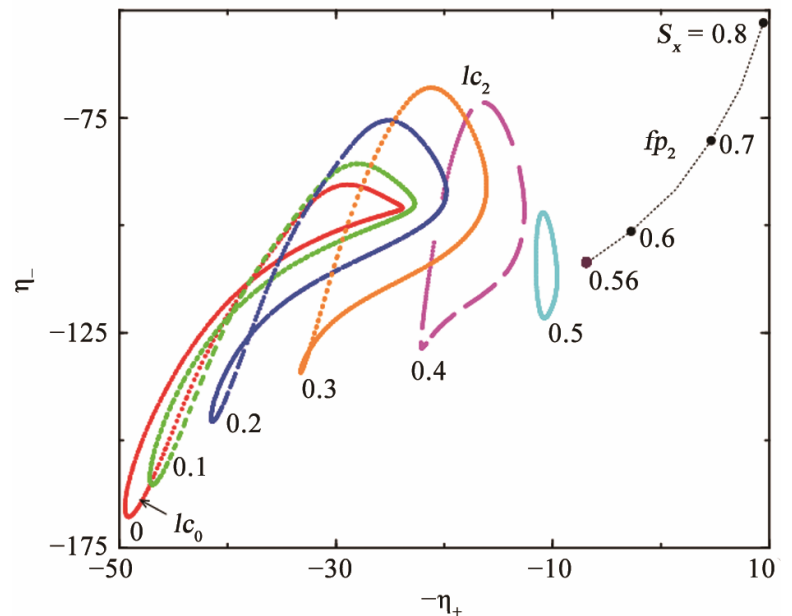

Figure 8. Phase portraits for $\mathrm{lc}_{0}, \mathrm{lc}_{2}$ and $\mathrm{fp}_{2}$ on $(\eta-,-\eta+)$ at different $s_{x}$ as indicated.

over time. For visual propose we will separate the momentum flux into its both contributions

$$
J \text { dif }:=r^{3}\langle u v / r\rangle_{a(r)} \text { and } J a d v:=-r^{3} v \partial_{r}\langle u v / r\rangle_{a(r)}
$$

(c.f. Equation (10)) characterizing diffuse and advective part respectively. Additional we will also normalize the momentum flux with the corresponding CCF angular momentum flux $J^{c f f}$ for $s_{x}=0$. Hence we only use the CCF-normalized quantities $J^{N}:=J / J^{\text {cff }}$,

$$
J^{N} \text { dif }:=J \text { dif } / J^{c f f} \text { and } J^{N} a d v:=J a d v / J^{c f f} \text {. }
$$

The spacetime plots in Figure 9 show either the full angular momentum flux $J^{N}$ and separate its both components $J^{N} d i f$ and $J^{N} a d v$ for $\mathrm{lc}_{0}$ in absence of a magnetic field. Note that this flow is axisymmetric. In addition, the averaged azimuthal velocity $\langle v\rangle_{a(r)}$ is also shown.

The full momentum flux $J^{N}$ is dominated by the diffu- 

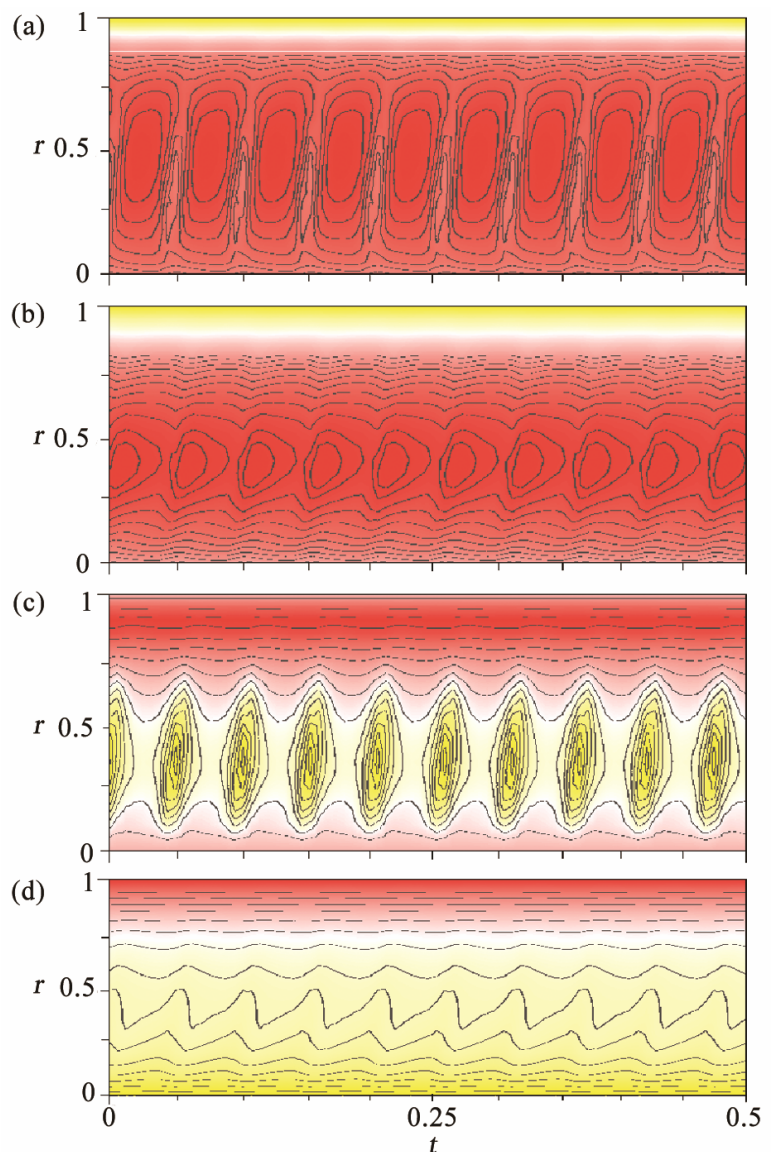

Figure 9. Spacetime plot $J^{N}:=J / J^{c f f} \quad$ (a) (c.f. Equation (9)) and its split up into the both contributions

$J^{N}$ dif $:=J d i f / J^{c f f} ;$ (b) and $J^{N} a d v:=J a d v / J^{c f f}$ (c) as averaged azimuthal velocity $\langle v\rangle a(r)$ (d) for $\mathrm{Ic}_{0}$ at $s_{x}=0$. Red (yellow) indicates positive (negative) values. Contours are shown with $\Delta J^{N}=\Delta J^{N} d i f=\Delta J^{N} a d v=0.2$ and for $\Delta\langle v\rangle a(r)=\mathbf{5 0}$.

sive contribution $J^{N}$ dif. Thereby its both contributions $J^{N} d i f$ and $J^{N} a d v$ show variations over one period just alternating to each other. While $J^{N}$ dif varies almost harmonic over one period $J^{N} a d v$ shows more pronounced dynamics.

For first half of the period it is almost constant before it fast increases and then significantly decreases in the second half of each period.

Comparing the momentum flux for $\mathrm{lc}_{2}$ at $s_{x}=0.4$ (c.f Figure 10) with that for lco at $s_{x}=0$ (c.f. Figure 9) one observes the shape in all spacetime plots to be quite similar. Merely the modulations for $\mathrm{lc}_{2}$ become weaker.

This holds for either the full angular momentum flux $J^{n}$ and its both contributions $J^{N}$ dif and $J^{N} a d v$ and for the averaged velocity $\langle v\rangle_{a(r)}$ in similar way. At the bifurcation point of $\mathrm{lc}_{2}$ at $s_{x}$ about 0.553 the time-dependence of all quantities vanish.
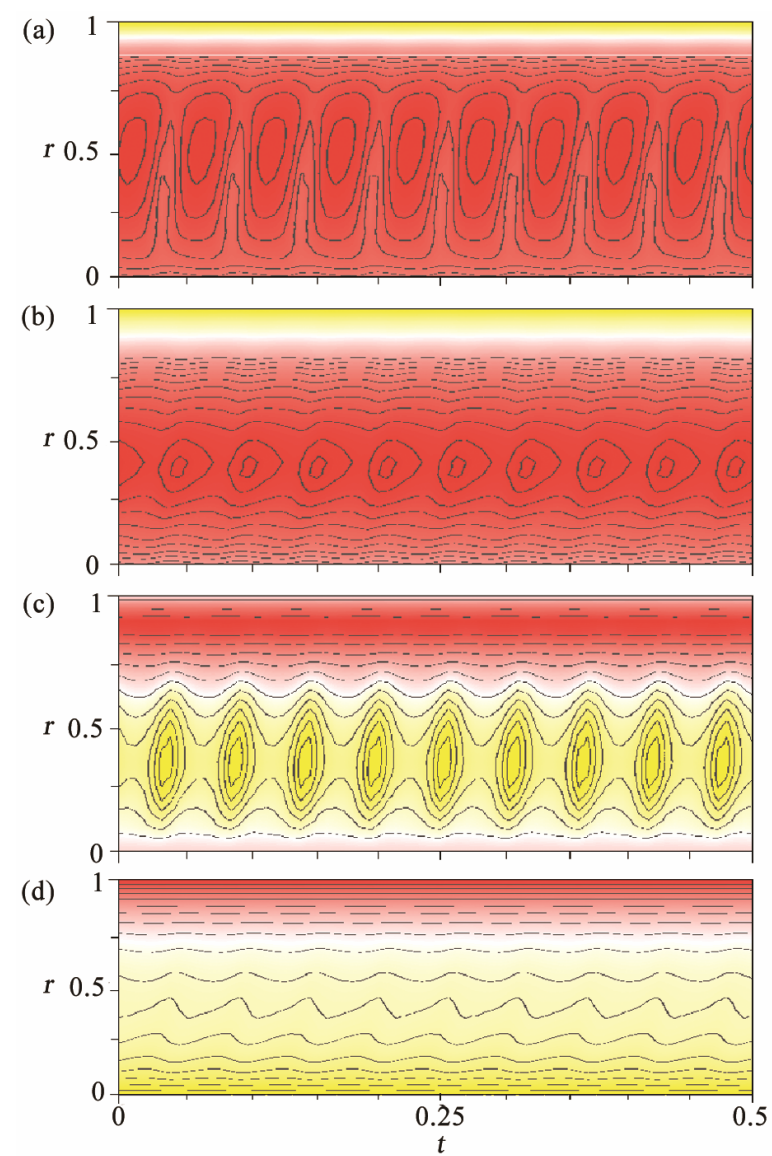

Figure 10. As Figure 9 but for $\mathrm{Ic}_{2}$ at $s_{x}=0.4$. Contours are shown with $\Delta J^{N}=\Delta J^{N}$ dif $=\Delta J^{N} a d v=0.2$ and for $\Delta\langle v\rangle a(r)=\mathbf{5 0}$.

Even while the dominant dynamics starts at the inner cylinder boundary layer due to the two streams along the inner cylinder (from both end-walls to mid-height) to merge in an outward directed jet the angular momentum flux do not show any significant modifications in this region. Its variations are largest in the bulk interior over a relative wide radial gap (c.f. Figures 9(a) and 10(a)).

Only the advective component that also shows the largest variations indicates a slightly orientation towards the inner boundary layer.

The temporal evolution of radial averaged momentum flux $\left\langle J^{N}\right\rangle_{r}$ is presented in Figure 11. Obviously the diffusive part $\left\langle J^{N} \text { dif }\right\rangle_{r}$ dominates the full momentum flux. But while this is almost constant in time the minor advective part $\left\langle J^{N} a d v\right\rangle_{r}$ shows significant periodic timedependence effecting the full momentum flux visible in the modulation by time. Comparing $\left\langle J^{N} \text { dif }\right\rangle_{r}$ with the long-time averaged momentum flux $\left\langle J^{N}\right\rangle_{r, t}$ (horizontal dashed lines) reflects its dominance. Note that Figure 11 shows the absolute magnitudes of the momentum flux and its contributions. $J^{N} a d v$ is negative 

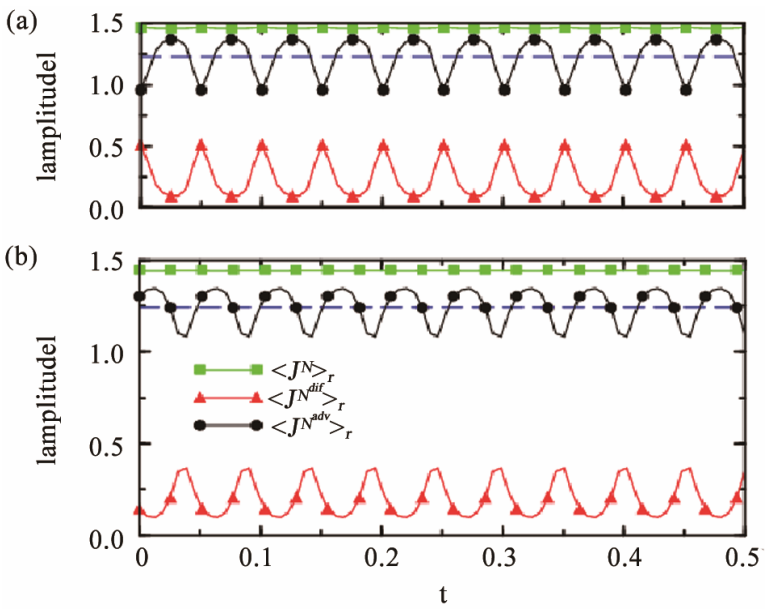

Figure 11. Variation with $t$ for absolute magnitudes $\left\langle J^{N}\right\rangle_{r}$ and its both contributions $\left\langle J^{N} \text { dif }\right\rangle_{r}$ and

$\left\langle J^{N} a d v\right\rangle_{r}$ for $\mathrm{Ic}_{0}$ at $s_{x}=0$ (a) and $\mathrm{Ic}_{2}$ at $s_{x}=0.4$

corresponding to Figures 8 and 9. Horizontal dashed lines indicate the long-time average $\left\langle J^{N}\right\rangle_{r, t}$ (c.f. Figure 12).

and therefore opposite directed to $\left\langle\boldsymbol{J}^{N} \text { dif }\right\rangle_{\boldsymbol{r}}$ minimizing the complete momentum flux $\mathrm{J}$.

Figure 12 shows the long-time average of momentumflux $\left\langle J^{N}\right\rangle_{r, t}$ (c.f. dashed lines in Figures 8-10) with $s_{x}$ and likewise the long-time and radial average azimuthal velocity $\langle v\rangle_{r, t}$. Both quantities decrease monotonously with $s_{x}$ until reaching the bifurcation threshold of $\mathrm{lc}_{2}$. Hereafter for $\mathrm{fp}_{2}$ the momentum flux do not virtually show any further variation-is more or less stagnated while $\langle v\rangle_{r, t}$ shows an almost linear decrease for further increase of $s_{x}$.

The almost constant value $\left\langle J^{N}\right\rangle_{r, t}$ for $s_{x}>0.553$ might be explained as in steady state $\mathrm{fp}_{2} \Delta J a d v$ becomes "neglectable". Thus only small modifications in the diffusive component due to variation of $s_{x}$ change the momentum flux $\left\langle J^{N}\right\rangle_{r, t}$ But as for $\mathrm{lc}_{2}$ these modifications are quite small. The radial position of the maximal modifications $J_{\max }$ moves slightly outwards with increasing the field strength $s_{x}$. The visible slight tilting in all spacetime plots of $\mathrm{J}$ (c.f. circular contour lines, slightly tilted from bottom left to top right in Figures 9 and 10) indicate the dynamics to start at the inner cylinder boundary layer and from there entering into the bulk.

The energy content in transverse velocity component at radial distance $r$ and an instant of time can be measured by the so-called cross-flow energy,

$$
E^{c f}:=\left\langle u^{2}+w^{2}\right\rangle_{a(r)}
$$

(a)

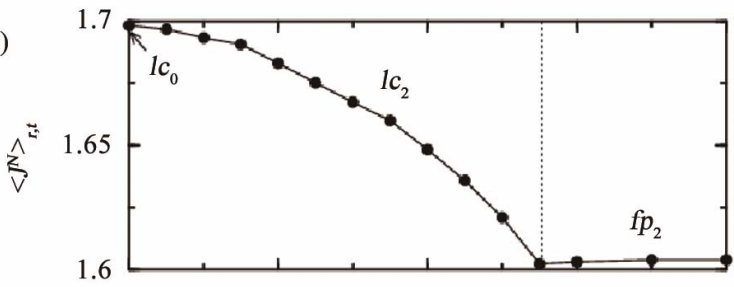

(b)

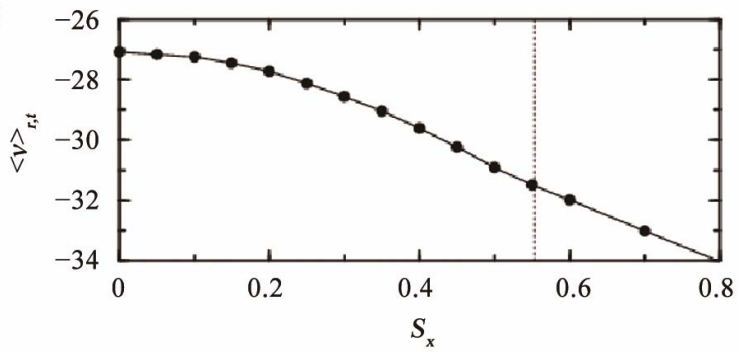

Figure 12. Variation with $s_{\mathrm{x}}$ of $\left\langle J^{N}\right\rangle_{r, t}$ (a) and $\langle v\rangle$ (b).

Again dotted lines indicate the bifurcation threshold for $\mathbf{l c}_{2}$.

As before $a(r)$ stands for areas averaged over the surface of cylinder at radius $r$.

Opposite to the angular momentum flux the cross-flow energy clearly indicates the region of largest modulation near the inner cylinder boundary layer (c.f. Figure 13), independent of the presence of a magnetic field is applied or not. The temporal modifications are stronger in absence of a magnetic field. As the momentum flux also the cross-flow energy becomes constant below the onset of $\mathrm{fp}_{2}\left(s_{x}>0.553\right)$. But other than the momentum flux the spacetime plot of cross-flow energy indicates two bands of regions where it is significant increased. A second dominant one also lying near the inner cylinder that coincide with the region where the flow is along the inner boundary layer starting near the lids to merge with the oscillating radial outward directed jet of angular momentum. Additionally a second weaker band of local increased cross-flow energy is visible arranged between mid-gap and quarter-half of the gap $0.5<r<0.75$.

As the $m=2$ contributions do not rotate the crossflow energy $E^{c f}$ is almost unaffected by variations of the field strength as visible in the almost identical shape of the cross-flow energy $E^{c f}$ for $\mathrm{lc}_{0}$ at $s_{x}=0$ and $\mathrm{lc}_{2}$ at 0.4 (c.f. Figure 13). The differences mainly result from the increase of $m=2$ contributions with $s_{x}$.

\section{Conclusion and Discussion}

In this paper, we investigated nonlinear hydrodynamics of time-dependent flow of a rotating ferrofluid. Therefore we considered a setup of differentially counter-rotating cylinders with small-aspect-ratio and wide-gap annulus and applied symmetry-breaking transverse magnetic fields. We found the flow can be driven from time-independent steady fixed-point solution to time-dependent, 

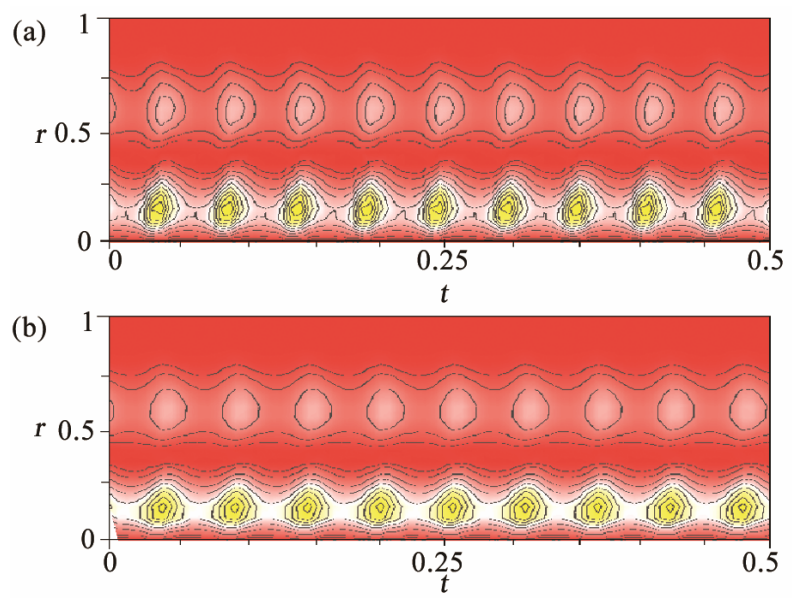

Figure 13. Space-time plot of the cross-flow energy, $E^{c f}(r, t)=\left\langle u^{2}+w^{2}\right\rangle a(r)$, averaged over the surfaces of a concentric cylinder for each radius $r$ for $l c_{0}$ at (a) $s_{x}=0$ and $\mathrm{lc}_{2}$ (b) $s_{x}=0.4$. Red (yellow) indicates high (low) energy with contours $\Delta E^{c f}=50$. Maximal energies are (a) 488.97 and (b) 424.47 (c.f Figures 9 and 10).

periodic limit-cycle solution and vice versa by only changing the strength of the applied magnetic field.

Due to shortness of our considered setup so-called anomalous mode solution exists in the system for not to high Reynolds numbers. In absence of a magnetic field these steady states are axisymmetric and exist symmetry-related. We elucidated this states also to exist in magnetic fields with finite transverse component. Even while they also exist symmetry related there axisymmetry is lost due to stimulation of finite $m=2$ contribution $[10,19]$. This mode-two symmetry is also preserved and underlying the time-periodic limit-cycle solution bifurcating out of these anomalous modes.

The time-independent flows show either a left-winding or right-winding helical shape due to the $m=2$ contributions [42] which do not rotate in azimuth-they are phase-pinned. Physically one observes two "bellies" one on that side where the magnetic field enters the annulus and a second one on the opposite side where it exits the annulus again. Instead we found time-dependent limitcycle solution to include both contributions. In particular it shows a kind of stroboscoping over one period alternating between both left- and right-winding $m=2$ contribution.

Even while the symmetries are significant modified due to a finite transverse magnetic field neither the angular momentum flux nor the cross-flow energy is significantly modified. Independent of the magnetic field strength the momentum flux is always dominated by its diffusive contribution which only shows small time-dependent variations over one period. In contrast, the minor advective contribution which is opposite directed to the diffusive one, it illustrates more pronounced variations over one period which is responsible for the time-dependence of the whole momentum flux.

We want to finish with the interesting but so far still open point of existence of hysteresis also for the bifurcation scenario of time-dependent flows as it was found for time-independent flows. This might be the germ and motivation of further numerical and experimental works.

\section{REFERENCES}

[1] G. I. Taylor, "Stability of a Viscous Liquid Contained between Two Rotating Cylinders," Philosophical Transac tions of the Royal Society A, Vol. 223, No. 605-615, 1923, pp. 289-343.doi:10.1098/rsta.1923.0008

[2] R. Tagg, "The Couette-Taylor Problem," Nonlinear Science Today, Vol. 4, No. 3, 1994, pp. 1-25.

[3] P. Chossat and G. Iooss, "The Couette-Taylor Problem," Springer, Berlin, 1994. doi:10.1007/978-1-4612-4300-7

[4] R. C. DiPrima and H. L. Swinney, "Instabilities and Transition in Flow between Concentric Rotating Cylinders," In: H. L. Swinney and J. G. Gollub, Eds., Hydrodynamic Instabilities and the Transition to Turbulence, Topics in Applied Physics, Vol. 45, Springer, Berlin, 1985, pp. 139180.

[5] C. D. Andereck, S. S. Liu and H. L. Swinney, "Flow Regimes in a Circular Couette System with Independently Rotating Cylinders," Journal of Fluid Mechanics, Vol. 164, 1986, pp. 155-183. doi:10.1017/S0022112086002513

[6] R. E. Rosensweig, "Ferrohydrodynamics," Cambridge University Press, Cambridge, 1985.

[7] J. E. Hart, "A Magnetic Fluid Laboratory Model of the Global Buoyancy and Wind-Driven Ocean Circulation: Analysis," Dynamics of Atmospheres and Oceans, Vol. 41, No. 2, 2006, pp. 121-138. doi:10.1016/j.dynatmoce.2006.03.001

[8] J. E. Hart and S. Kittelman, "A Magnetic Fluid Laboratory Model of the Global Buoyancy and Wind-Driven Ocean Circulation: Experiments," Dynamics of Atmospheres and Oceans, Vol. 41, No. 2, 2006, pp. 139-147. doi:10.1016/j.dynatmoce.2006.03.002

[9] M. I. Shliomis, "Effective Viscosity of Magnetic Suspensions," Soviet Journal of Experimental and Theoretical Physics, Vol. 34, 1972, p. 1291.

[10] S. Altmeyer, C. Hoffmann, A. Leschhorn and M. Lücke, "Influence of Homogeneous Magnetic Fields on the Flow of a Ferrofluid in the Taylor-Couette System," Physical Review E, Vol. 82, No. 1, 2010, Article ID: 016321. doi:10.1103/PhysRevE.82.016321

[11] M. Reindl and S. Odenbach, "Effect of Axial and Trans Verse Magnetic Fields on the Flow Behavior of Ferrofluids Featuring Different Levels of Interparticle Interaction," Physics of Fluids, Vol. 23, No. 9, 2011, Article ID: 093102. doi: $10.1063 / 1.3633341$

[12] M. Holderied, L. Schwab and K. Stierstadt, "Rotational Viscosity of Ferrofluids and the Taylor Instability in a Magnetic Field," The European Physical Journal, Vol. 70, 
No. 4, 1988, pp. 431-433. doi:10.1007/BF01312116

[13] A. N. Vislovich, V. A. Novikov and A. K. Sinitsyn, "Influence of a Magnetic Field on the Taylor Instability in Magnetic Fluids," Journal of Applied Mechanics and Technical Physics, Vol. 27, No. 1, 1986, pp. 72-78. doi:10.1007/BF00911123

[14] M. Niklas, "Influence of Magnetic Fields on Taylor Vortex Formation in Magnetic Fluids," Zeitschrift für Physik B Condensed Matter, Vol. 68, No. 4, 1987, pp. 493-501. doi:10.1007/BF01471080

[15] M. Niklas, H. Müller-Krumbhaar and M. Lücke, "TaylorVortex Flow of Ferrofluids in the Presence of General Magnetic Fields," Journal of Magnetism and Magnetic Materials, Vol. 81, No. 1-2, 1989, pp. 29-38. doi:10.1016/0304-8853(89)90225-4

[16] O. Ambacher, S. Odenbach and K. Stierstadt, "Rotational Viscosity in Ferrofluids," Zeitschrift für Physik B Condensed Matter, Vol. 86, No. 1, 1992, pp. 29-32. doi:10.1007/BF01323543

[17] J. Singh and R. Bajaj, "Couette Flow in Ferrofluids with Magnetic Field," Journal of Magnetism and Magnetic Materials, Vol. 294, No. 1, 2005, pp. 53-62. doi:10.1016/i.jmmm.2004.10.123

[18] A. Leschhorn, M. Lücke, C. Hoffmann and S. Altmeyer, "Stability of Circular Couette Flow of a Ferrofluid in an Axial Magnetic Field: Influence of Polydispersity," Physical Review E, Vol. 79, No. 3, 2009, Article ID: 036308. doi:10.1103/PhysRevE.79.036308

[19] S. Altmeyer, J. Lopez and Y. Do, "Influence of an Inhomogeneous Internal Magnetic Field on the Flow Dynamics of Ferrofluid between Differentially Rotating Cylinders," Physical Review E, Vol. 85, No. 6, 2012, Article ID: 066314. doi:10.1103/PhysRevE.85.066314

[20] S. Altmeyer, J. Lopez and Y. Do, "Effect of Elongational Flow on a Ferrofluid under Magnetic Field," Physical Review E.

[21] S. Altmeyer, A. Leschhorn, C. Hoffmann and M. Lücke, "Elongational Flow Effects on the Vortex Growth out of Couette Flow in Ferrofluids," Physical Review E, Vol. 87, No. 5, 2013, Article ID: 053010. doi:10.1103/PhysRevE.87.053010

[22] T. B. Benjamin, "Bifurcation Phenomena in Steady Flows of a Viscous Fluid. I. Theory," Proceedings of the Royal Society A, Vol. 356, No. 1696, 1978, pp. 1-26. doi:10.1098/rspa.1978.0028

[23] T. B. Benjamin, "Bifurcation Phenomena in Steady Flows of a Viscous Fluid. II. Experiments," Proceedings of the Royal Society A, Vol. 356, No. 1696, 1987, pp. 27-43. doi:10.1098/rspa.1978.0030

[24] K. A. Cliffe, J. J. Kobine and T. Mullin, "The Role of Anomalous Modes in Taylor-Couette Flow," Proceedings of the Royal Society A, Vol. 439, No. 1906, 1992, pp. 341-357. doi:10.1098/rspa.1992.0154

[25] S. Altmeyer, C. Hoffmann, M. Heise, J. Abshagen, A. Pinter, M. Lücke and G. Pfister, "End Wall Effects on the Transitions between Taylor Vortices and Spiral Vortices," Physical Review E, Vol. 81, 2010, Article ID: 066313. doi:10.1103/PhysRevE.81.066313
[26] H. Furukawa, T. Watanabe, Y. Toya and I. Nakamura, "Flow Pattern Exchange in the Taylor-Couette System with a Very Small Aspect Ratio," Physical Review E, Vol. 65, No. 3, 2002, Article ID: 036306. doi:10.1103/PhysRevE.65.036306

[27] T. B. Benjamin and T. Mullin, "Anomalous Modes in the Taylor Experiment," Proceedings of the Royal Society A, Vol. 377, No. 1770, 1981, pp. 221-249. doi:10.1098/rspa.1981.0122

[28] S. Altmeyer, Y. Do, F. Marques and J. Lopez, "Symmetry-Breaking Hopf Bifurcations to 1-, 2-, and 3-Tori in Small-Aspect-Ratio Counterrotating Taylor-Couette Flow", Physical Review E, Vol. 81, No. 4, 2010, Article ID: 066313. doi:10.1103/PhysRevE.86.046316

[29] K. A. Cliffe, "Numerical Calculations of Two-Cell and Single-Cell Taylor Flows," Journal of Fluid Mechanics, Vol. 135, 1983, pp. 219-233. doi:10.1017/S0022112083003055

[30] A. Schulz, G. Pfister, and S. J. Tavener, "The Effect of Outer Cylinder Rotation on Taylor-Couette Flow at Small Aspect Ratio," Physics of Fluids, Vol. 15, No. 2, 2013, pp. 417-425. doi:10.1063/1.1532340

[31] M. Nagata, "On Wavy Instabilities of the Taylor-Vortex Flow between Corotating Cylinders," Journal of Fluid Mechanics, Vol. 88, 1988, pp. 585-598.

[32] M. Cross and P. Hohenberg, "Pattern Formation outside of Equilibrium," Reviews of Modern Physics, Vol. 65, No. 3, 1993, pp. 851-1112. doi:10.1103/RevModPhys.65.851

[33] R. Hollerbach and A. Fournier, "End-Effects in Rapidly Rotating Cylindrical Taylor-Couette Flow," AIP Conference Proceedings, 2004, pp. 114-121.

[34] H. Ji, M. Burin, E. Schartman and J. Goodman, "Hydrodynamic Turbulence Cannot Transport Angular Momentum Effectively in Astrophysical Disks," Nature, Vol. 444, No. 7117, 2006, pp. 343-346. doi: $10.1038 /$ nature 05323

[35] F. Stefani, T. Gundrum, G. Gerbeth, G. Rüdiger, M. Schultz, J. Szklarski and R. Hollerbach, "Experimental Evidence for Magnetorotational Instability in a Taylor-Couette Flow under the Influence of a Helical Magnetic Field," Physical Review Letter, Vol. 97, No. 18, 2006, Article ID: 184502. doi:10.1103/PhysRevLett.97.184502

[36] M. Paoletti and D. Lathrop, “Angular Momentum Transport in Turbulent Flow between Independently Rotating Cylinders," Physical Review Letter, Vol. 106, No. 2, 2011, Article ID: 024501 doi:10.1103/PhysRevLett.106.024501

[37] D. van Gils, S. Huisman, G.-W. Bruggert, C. Sun and D. Lohse, "Torque Scaling in Turbulent Taylor-Couette Flow with Co- and Counter-rotating Cylinders," Physical Review Letter, Vol. 106, No. 2, 2011, Article ID: 024502. doi:10.1103/PhysRevLett.106.024502

[38] S. A. Balbus and J. F. Hawley, "Instability, Turbulence, and Enhanced Transport in Accretion Disks," Reviews of Modern Physics, Vol. 70, No. 1, 1998, pp. 1-53.

[39] S. A. Balbus, "Enhanced Angular Momentum Transport in Accretion Disks," Annual Review of Astronomy and Astrophysics, Vol. 41, 2003, pp. 555-597. 
doi:10.1146/annurev.astro.41.081401.155207

[40] P. Langevin, "Magnetisme et Theorie Deselectrons," Annales de Chemie et de Physique, Vol. 5, No. 7, 1905, pp. 70-127.

[41] J. Embs, H. W. Müller, C. Wagner, K. Knorr and M. Lücke, "Measuring the Rotational Viscosity of Ferrofluids without Shear Flow," Physical Review E, Vol. 61, No. 3, 2000, pp. R2196-R2199. doi:10.1103/PhysRevE.61.R2196

[42] H. W. Müller and M. Liu, "Structure of Ferrofluid Dynamics," Physical Review E, Vol. 64, No. 6, 2001, Article
ID: 061405. doi:10.1103/PhysRevE.64.061405

[43] M. Reindl and S. Odenbach, "Influence of a Homogeneous Axial Magnetic Field on Taylor-Couette Flow of Ferrofluids with Low Particle-Particle Interaction," Experiments in Fluids, Vol. 50, No. 2, 2011, pp. 375-384.

[44] B. Eckhardt, S. Grossmann and D. Lohse, "Flux and Energy Dissipation in Thermal Convection and Shear Flows," Europhysics Letter, Vol. 78, No. 2, 2007, Article ID: 24001. doi:10.1209/0295-5075/78/24001 